\title{
Construcción y validación de un instrumento multivariable para la evaluación de
}

\section{Calidad de Vida en ancianos}

\author{
Andrés I. Urrutia ${ }^{1}$, Livio T.P. Grasso **, \& Elena R. Guzmán* \\ */**Cátedra de Psicología Evolutiva del Adulto y la Senectud. \\ */**Facultad de Psicología, Universidad Nacional de Córdoba, Córdoba, Argentina. \\ **Cátedra de Psicoestadística.
}

Resumen. El El concepto de Calidad de Vida (CV), es un constructo multidimensional que se aleja del criterio clásico de salud ya que incluye la evaluación del sujeto según sus particulares estándares de satisfacción y bienestar. En este trabajo se presenta el Cuestionario Breve de Calidad de Vida (CBCV), el cual evalúa las diferentes dimensiones de la Calidad de Vida en adultos mayores. Este instrumento fue aplicado a 512 adultos mayores de la ciudad de Córdoba. Los resultados muestran que es elevada la fiabilidad de cada uno de las dimensiones que este cuestionario evalúa. Se realizaron análisis factoriales para cada dimensión de la CV, permitieron conformar un instrumento abreviado con muy buenas características psicométricas. Por lo tanto este constituye un instrumento útil para la valoración de las necesidades particulares de los mayores, pudiendo ser utilizado en la planificación y oferta de servicios socio-sanitarios.

Palabras clave: Calidad de Vida, Ancianidad, Psicogerontología, Salud, Bienestar Psicológico.

Title: Construction and validation of a multivariable instrument for assessing quality of life in elderly.

Abstract: The concept of Quality of Life $(\mathrm{QoL})$ is a multidimensional construction that differs from the traditional criteria of health since it includes an evaluation of individuals according to his particular standards of satisfaction and wellbeing. This article introduces a Brief Questionnaire of Quality of Life (CBCV) that evaluates the different dimensions of Quality of Life in elder people. This instrument was applied to a sample of 512 seniors in the city of Cordoba, Argentina. The results demonstrate high reliability on each dimension that this questionnaire evaluates. Factor analysis were carried out for each dimension of the QoL, allowed us to develop an abbreviated tool with satisfactory psychometric properties. Therefore, this is a useful instrument for particular needs of the elderly people assessment, which could be used in the design and provision of socio-sanitary services.

Key Words: Quality of life, Elderly, Psychogerontology, Health, Psychological wellbeing.

\section{Introducción}

El envejecimiento hace referencia a una realidad multifacética atravesada $\mathrm{y}$ determinada no sólo por aspectos cronológicos, sino también por aspectos fisiológicos, legislativos, sociales y culturales. Cada sociedad establece los límites de edad a partir del cual una persona se considera perteneciente a la ancianidad, por lo cual la definición de la vejez se encuentra determinada socioculturalmente.

Aunque la frontera que determina el inicio de la vejez ha ido variando a lo largo de los tiempos, en la actualidad, la mayoría de los países de la región en sus respectivas

\footnotetext{
${ }^{1}$ Por favor dirigir la correspondencia relacionada con este artículo a: Dr. Andrés I. Urrutia, Viamonte 76, Barrio General Paz, Córdoba, Argentina Tel.: 0054-351-452 40 77. E-mail: urrutias@infovia.com.ar

Lic. Elena R. Guzmán, Rivadavia 720, Dpto, D Barrio Centro, Córdoba, Argentina Tel: 0054-0351-425 1399 , E-mail: elenarguzman@gmail.com
} 
legislaciones, ha establecido un criterio cronológico de inicio de la vejez, considerando el comienzo de la misma a partir de los 60 años (CEPAL-CELADE, 2006).

En la actualidad, el aumento de la expectativa de vida combinado con la disminución en las tasas de natalidad es sin duda resultado del vertiginoso desarrollo de la tecnología médica y farmacológica, las cuales se encuentran en un constante avance determinando un incremento en el número de personas mayores en la población mundial.

No obstante, pese a estos considerables adelantos, gran parte de los adultos mayores pueden presentar limitaciones físicas, funcionales, así como daños orgánicos o psicológicos que pueden provocar una pérdida de la autonomía excluyéndolos paulatinamente de sus redes sociales.

Actualmente gran parte de las sociedades europeas se encuentran "envejecidas", esto también puede observarse en algunos países de América Latina como la república Argentina, Uruguay y Cuba. Este aumento en la cantidad de personas mayores está acompañado por el aumento de necesidades y uso de servicios sociales y de salud.

Por lo tanto, el envejecimiento se perfila no solo como un fenómeno poblacional, sino también como una problemática social que merece el desarrollo de procedimientos que puedan garantizar cierta equidad intergeneracional en la distribución de los recursos. Es esperable que el colectivo de los ancianos como tal pueda contar con los cuidados y servicios de salud deseados, los cuales usualmente difieren de los que el estado está dispuesto a ofrecerles.

Los indicadores de salud usados habitualmente para la evaluación del sistema de salud, se encuentran más ligados a enfermedad y muerte constituyendo una expresión reducida de la salud y son insuficientes para describir la calidad de vida de los individuos. No se trata ya de vivir más, sino de vivir mejor. De tal manera, se requieren otras condiciones e indicadores psicológicos y sociales a la hora de establecer la salud de una comunidad o grupo social, particularmente en los adultos mayores.

Es necesario realizar una valoración exhaustiva de la capacidad de funcionamiento de cada una de las áreas que determinan que una persona mayor pueda llevar una vida autónoma, lo cual implica: salud física y mental, capacidad para desarrollar actividades de la vida cotidiana, recursos económicos y soporte social.

La salud, como el cuidado de la misma, son obviamente componentes importantes de la calidad de vida, pero no comprenden las condiciones necesarias y suficientes para ser en sí 
mismas un concepto amplio y holístico, ya que deja por fuera un sinnúmero de factores psicológicos y sociales de singular importancia.

El concepto de Calidad de Vida (CV) surgió en consonancia con el concepto de salud definido por la Organización Mundial de la Salud (OMS, 1994): "un estado de completo bienestar, físico, psíquico y social". Adicionalmente, la calidad de vida pone en consideración tanto los factores físicos, psíquicos y sociales de la salud, así como la particular valoración subjetiva que los sujetos realizan de las diferentes dimensiones de su vida.

Aunque dicho concepto actualmente ha logrado el reconocimiento y la aceptación general, ha sido difícil acordar una definición universal del mismo. La Organización Mundial de la Salud, en el año 1994, propuso una definición de consenso de calidad de vida, considerándola como "percepción personal de un individuo de su situación en la vida, dentro del contexto cultural y de valores en que vive, y en relación con sus objetivos, expectativas, valores e intereses". (OMS, 1994)

La calidad de vida es un concepto amplio que incluye no solamente el estado de salud, aspectos económicos, medio ambientales y educativos, sino también aspectos legislativos y aquellos relativos a los sistemas de salud (Yanguas Lezaun, 2006). En este sentido, los autores Birren y Diekmann (1991) definen a la CV como: "la evaluación de las circunstancias de la vida de un individuo, grupo o población, en la cual se evalúan las características del ambiente físico y social, los estados internos del sujeto y su salud; presentándose dos posibles modos de medición: uno basado en la percepción subjetiva o interna de la CV y otro a través de juicios externos y valoraciones objetivas de la misma”.

Lawton (1991) amplía esta definición al contemplar los aspectos objetivos y subjetivos de la evaluación de la calidad de vida, incluyendo tanto la medición de la salud física, funcional, el estado mental, el funcionamiento cognitivo, la existencia de redes sociales, el nivel socio-económico-educativo, como los determinantes ambientales (subjetivos y objetivos), la percepción del estado de salud, la satisfacción vital, la autoestima, y las diferentes medidas de bienestar subjetivo. Para este autor, la CV es: "la evaluación multidimensional, tanto en criterios de norma o de consenso social, como la experiencia individual, del sistema persona-ambiente, tanto en un tiempo presente, como en el pasado y en su perspectiva futura".

La utilidad de la evaluación del atributo de la Calidad de Vida (CV) se basa en la posibilidad de estimar los beneficios reales de los tratamientos -tanto individuales cuanto 
grupales- y los costos sociales de dichas intervenciones, teniendo en cuenta los derechos de los adultos más ancianos para controlar la forma en que viven y mueren.

Existen diferentes instrumentos que evalúan la $\mathrm{CV}$, lo que demuestra un creciente interés en la posibilidad de indagar las diferentes dimensiones que la componen. Estas pruebas suelen ser extensas y usualmente requieren de investigadores entrenados para su administración. Por otra parte, los instrumentos cortos, generalmente de autoaplicación, usualmente son unidimensionales. Es, por lo tanto, un desafío el desarrollar un instrumento que sea exhaustivo, que considere las dimensiones esenciales, pero de fácil administración y de una duración moderada.

El Cuestionario de Calidad de Vida en la III Edad desarrollado por la Dra. Rocío Fernández Ballesteros y sus colaboradores (Fernández Ballesteros, 1996) ha sido utilizado en el trabajo "Calidad de Vida de la Vejez en Distintos Contextos", en el marco de un convenio de la Universidad Autónoma de Madrid y el INSERSO (Instituto Nacional de Servicios Sociales), instrumento sobre el cual se generó el CBCV, adaptándolo a la población local.

El valor del presente instrumento radica en que realiza una evaluación multidimensional de la Calidad de Vida de sujetos de la tercera edad (que incluye salud, habilidades funcionales, actividades de ocio y tiempo libre, relaciones interpersonales, calidad de los servicios sociales y de salud, calidad ambiental en el propio hogar y en el entorno y el nivel de ingresos) contemplando tanto las dimensiones objetivas como subjetivas de estos aspectos.

A partir del Cuestionario de Calidad de Vida presentado, los mismos autores elaboraron un Cuestionario Breve de Calidad de Vida (CUBRECAVI). En éste se consideraron cinco variables relevantes o criteriales a la hora de establecer el poder discriminatorio de las respuestas de los sujetos. La fiabilidad o consistencia interna del instrumento dio como resultado índices altos de fiabilidad, ya que arrojaron un resultado por encima de $\alpha=.70$.

Por tanto, el objetivo de este trabajo es presentar la construcción de una prueba que considere la multidimensionalidad de la $\mathrm{CV}$, que provea evidencias relacionadas a la validez del constructo, y logre constituirse en un instrumento local de referencia para la evaluación de los servicios ofrecidos a los mayores. Ya que, hasta que las diferencias subjetivas, sociales y culturalmente originadas, e históricamente determinadas no se incluyan en las formas de definir la calidad de vida, los servicios y prestaciones resultarán inapropiados, insuficientes, o 
al menos, irrespetuosos de las diferencias constitutivas de los sujetos mayores.

\section{Método}

\subsection{Participantes.}

La muestra estuvo constituida por 515 personas mayores que fueron divididos en dos sub-muestras. La primera de ellas se integró con sujetos asistentes a Centros Diurnos Municipales (CDM), los que fueron escogidos proporcionalmente según el número total de asistentes en cada centro, (muestreo estratificado); los sujetos de cada institución fueron seleccionados a través de un muestreo aleatorio simple, de 1078 ancianos y se incluyeron en la muestra 324 ancianos (31\% del total). La submuestra 2 se conformó por 191 ancianos residentes en geriátricos. Primeramente se escogieron al azar 40 residencias geriátricas de un total de 80 , de las que en cada una se extrajeron en forma aleatoria de 4 a 12 ancianos, según el número de residentes de cada una.

La muestra incluyó 420 mujeres $(81,6 \%)$ y 95 varones $(18,4 \%)$. Para la submuestra 1 (asistentes a Hogares de Día Municipales), la media de edad fue de 69,84 años, con una desviación típica de 7,04, mientras que para la sub-muestra 2 (residentes en geriátricos), la media fue de 79,017, con una desviación típica de 8,37 años. La mayoría de los participantes eran viudos $(54,6 \%)$, en menor medida casados o en pareja $(23,2 \%)$ y solteros, separados o divorciados $(22 \%)$.

En relación al nivel escolar, el 32.9\% de los de los participantes no tenían estudios, el $33.7 \%$ realizó estudios primarios, el $14.1 \%$ no completó el nivel medio y el $19.3 \%$ concluyó estos estudios. En cuanto al grupo de convivencia, el 18,3\% vivían solos, el 14\% solo con su pareja, el $24,4 \%$ con un grupo familiar extenso (pareja, hijos y nietos), el 35,1\% vivían en residencias y el 8,2\% lo cohabitaban con familiares indirectos o sin lazos sanguíneos.

\subsection{Instrumentos.}

El Cuestionario Breve de Calidad de Vida (CVCB) consta de 180 ítems agrupados de acuerdo a la definición multidimensional de Calidad de Vida expresada por Lawton (1991) en las siguientes dimensiones: (1) Estado mental, (2) Salud física objetivamente valorada, (3) Evaluación subjetiva de la salud, (4) Integración social, (5) Nivel funcional, (6) Utilización del tiempo y nivel de actividad, (7) Calidad ambiental, y (8) Satisfacción vital.

Los ítems correspondientes a la dimensión Estado mental, apuntan a determinar la presencia de trastornos cognitivos asociados a demencias o daños neurológicos específicos y la 
dimensión de Salud física objetivamente valorada, incluye índices de enfermedad, trastornos crónicos y dolores recientes. Se valora también la ingesta medicamentos, las visitas al médico, las internaciones o necesidad de reposo. La Evaluación subjetiva de la salud indaga el grado en el cual los ancianos consideran su estado de salud, el nivel de satisfacción con la misma, comparación de su salud con la de otras personas de su edad, la autovaloración del declive de su salud y de la evolución de la misma. Dentro de la dimensión Integración social se indagan los contactos y la frecuencia de visitas según el tipo de vínculo (hijos/nietos) y el grado de satisfacción que dichas interacciones producen. Adicionalmente se incluyen ítems que evalúan la presencia, frecuencia y satisfacción de las relaciones sexuales, así como el estado y modificación de la apetencia sexual. Nivel funcional, valora el grado de dificultad en las conductas autónomas y sirve como indicador del deterioro o mantenimiento de la salud. Para analizar esta dimensión se incluyen ítems relacionados a las actividades básicas de la vida diaria, así como de las actividades instrumentales de la vida diaria. Se incluye también: la valoración subjetiva del nivel funcional pidiéndoles comparación con el de otras personas de su edad, la valoración del declive funcional y de la perspectiva evolución del mismo en un futuro. La dimensión de Utilización del tiempo y nivel de actividad, mide la frecuencia de realización de diversas actividades y la valoración subjetiva de la misma se valora a través la evaluación del grado de satisfacción que la realización de las mismas actividades les produce. Se estima el grado de satisfacción de los ancianos con la Calidad ambiental en cuanto las condiciones objetivas de su vivienda y su barrio. Para la dimensión Satisfacción vital se incluyen ítems que evalúan el agrado o disgusto con su propio estado actual, el nivel de optimismo hacia el futuro, el deseo de vivir el pasado en lugar del presente y el grado de satisfacción con la vida.

\subsection{Procedimiento.}

Se entrenaron a profesionales para la recolección de datos, cuidando que realizaran entrevistas a distintos centros de aquellos en los que trabajaban para garantizar la objetividad en la toma del cuestionario. Las entrevistas tuvieron una duración promedio de 90 minutos aproximadamente, dependiendo de la motivación, nivel cognitivo y de comprensión de las consignas por parte de los ancianos. Se excluyeron del estudio aquellos ancianos que padecían una demencia.

Se realizó un análisis factorial exploratorio en cada una de las dimensiones, así como de la escala completa, con el método de extracción de componentes principales con rotación 
Varimax. Los factores se seleccionaron utilizando dos criterios: la regla de Kaiser-Gutman (K1): factores con autovalor igual o mayor a 1 y se consideró para la selección de los mismos el método gráfico de análisis de la pendiente de Cattel (Scree test).

A partir del análisis de la matriz de factores rotados, se eliminaron los ítems cuyos pesos factoriales fueran menores a .40 , aquellos con cargas compartidas con otro factor superior a .30 (ítems complejos) y finalmente se eliminaron aquellos ítems que no saturaban en ningún factor. Los mismos criterios y procedimientos para la inclusión o exclusión de los ítems en las sub-escalas fueron seguidos a la hora de realizar el análisis para todo el instrumento.

\section{Resultados}

\subsection{Análisis factorial.}

Del análisis factorial de los diez elementos que componen el Estado mental (ver Tabla 1) se extrajeron dos factores, los cuales en conjunto explican un 49,2\% de la varianza total. El primer factor explica un $37,3 \%$ de la varianza y se lo denomina "Información /Actualidad" ya que en él saturan los ítems que se encuentran relacionados con conocimientos cotidianos y sociales: nombres de presidentes, ministros, resta seriada, dirección de la personas y fecha en que se realiza la evaluación (ítems 6, 3, 1, 7, 4, 5 y 10). El Factor 2 explica un 12\% de la varianza, al cual se denominó "Información Primaria”. En el saturaron los ítems que valoran datos ligados a información registrada muy tempranamente (apellido materno, color de la bandera y nombre de la ciudad).

El análisis de la Salud subjetiva (ver Tabla 2) arrojó una solución de 2 factores que explica el $60 \%$ de la varianza total. El Factor 1 explica por sí mismo un 39,4\% de la varianza y podría identificarse como "Valoración Actual" del estado de salud, ya que en él saturaron ítems que suponen una evaluación de cómo ha evolucionado la salud hasta la fecha y cómo se encuentra hoy. El Factor 2 "Evolución temporal de la salud" toma en consideración la expectativa de funcionamiento y parámetros comparativos. Este factor explica el $21 \%$ de la varianza. 
Tabla 1.

Matriz de factores rotados de los ítems de la sub-escala de estado mental

\begin{tabular}{lcc}
\hline Factor & Factor 1 & Factor 2 \\
\hline Nombre del ministro de economía & .721 & \\
Nombre del barrio & .716 & \\
Fecha de hoy & .704 & \\
Nombre del presidente anterior & .675 & \\
Dirección & .645 & \\
Nombre del presidente & .592 & \\
Resta seriada de 3 en 3 empezando desde el 20 & .510 & .771 \\
Color de la bandera & & .747 \\
Nombre de la ciudad & & .616 \\
Apellido materno & & 1.92 \\
\hline \multicolumn{1}{c}{ Autovalor } & 3.73 & 11,49 \\
\hline explicado de la varianza & 37,3 & \\
\hline
\end{tabular}

Tabla 2.

Matriz de factores rotados de los ítems de la sub-escala de salud subjetiva

\begin{tabular}{lcc}
\hline Factores & Factor 1 & Factor 2 \\
\hline Satisfacción con la salud actual & .832 & \\
Valoración de la salud actual & .794 & \\
Salud respecto a otros & .553 & \\
Salud en el año próximo & & .897 \\
Salud actual comparada con el año anterior & .476 & .572 \\
\hline Autovalor & 1.96 & 1.06 \\
\% explicado de la varianza & 39,4 & 21,4 \\
\hline
\end{tabular}

El análisis de la sub-escala Padecimiento de enfermedades e ingesta de medicamentos (ver Tabla 3) arrojó una solución factorial de 3 factores que explican un 24,6\% de la varianza total. El Factor 1 se denominó "Problemas osteo articulaes" ya que saturaron ítems relacionados a dolores de huesos y articulaciones, dolores e hinchazón de pies, reuma, cansancio sin razón aparente y hormigueo en brazos y piernas. El segundo factor se denominó "Trastornos del sueño", ya que en él saturaron principalmente el insomnio, la dificultad para dormir o conciliar el sueño en los últimos 15 días y la ingesta de pastillas para dormir o tranquilizantes. Este factor explica un 5,7\% de la varianza. El Factor 3 se denominó "Problemas bronquio-respiratorios" ya que todos los ítems que saturaron en él se refieren a 
trastornos crónicos u ocasionales relacionados con las vías respiratorias o su tratamiento. Este tercer factor explica un $4,5 \%$ de la varianza.

\section{Tabla 3.}

Matriz de factores rotados de los ítems de las sub-escalas: padecimiento de enfermedades e ingesta de medicamentos

\begin{tabular}{lccc}
\hline Ítems & Factor 1 & Factor 2 & Factor 3 \\
\hline Dolor de huesos, columna o articulaciones en los últ. 15 días & .688 & \\
Reuma, artrosis, lumbalgia o dolor de espalda en el último año & .669 & \\
Cansancio sin razón aparente en los últimos 15 días & .599 & \\
Tobillos hinchados en los últimos 15 días & .588 & \\
Hormigueo en brazos y piernas en los últimos 15 días & .551 & \\
Problemas persistentes en los pies en el último año & .533 & \\
Medicinas para el reuma & -.500 & \\
Ingesta de antibióticos en los últimos 15 días & -.500 & \\
Mareos o vahídos en los últimos quince días & .497 & & \\
Estreñimiento en el último año & .445 & & \\
Insomnio en el último año & & .727 & \\
Dificultad para dormir en los últimos 15 días & & .706 & \\
Ingesta de tranquilizantes en los últimos 15 días & &. .692 & \\
Problemas cardíacos y circulatorios & & .480 & \\
Ingesta de antidepresivos en los últimos 15 días & & -.470 & \\
Medicamentos para corazón o circulación en los últ. 15 días & & -.454 & \\
Tensión arterial alta en el último año & & & \\
Ingesta de antigripales y antihistamínicos en los últimos 15 d. & & & \\
Tos, catarro o gripe en los últimos 15 días & & &. .713 \\
Bronquitis asma en el último año & & & .644 \\
Ingesta de antibióticos en los últimos 15 días & & & \\
Dificultad para respirar o falta de aire en los últimos 15 días & & & \\
Ingesta de antifebriles en los últimos 15 días & & & \\
Dolor u opresión en el pecho en los últimos 15 días & & & \\
Palpitaciones en los últimos 15 días & & & \\
\hline Autovalor & & & \\
\% explicado de la varianza & & & \\
\hline
\end{tabular}

Como resultado del análisis factorial de la dimensión Salud psíquica (ver Tabla 4), se obtuvieron dos factores que explican un $43.9 \%$ de la varianza total. El Factor 1 explica el $32 \%$ de la varianza y refleja Problemas mnésicos en diferentes grados, desde olvidos benignos y distracciones hasta trastornos que comprometen más seriamente el nivel cognitivo. El Factor 2 se lo denominó Neuroticismo ya que sobre él saturan todos los ítems relacionados a malestares emocionales y subjetivos específicos (estar deprimido, sentirse 
observado, nervioso o, tener ideas fijas o llorar y reír sin motivo). Este factor explica un $11.8 \%$ de la varianza.

Tabla 4.

Matriz de factores rotados de los ítems de la sub-escala de salud psíquica

\begin{tabular}{lcc}
\hline Ítems & Factor 1 & Factor 2 \\
\hline Olvidarse de las cosas que hizo ayer & .816 & \\
Olvido del día de la semana o lo que ha estado haciendo & .730 & \\
Olvidar dónde puso sus objetos personales & .691 & \\
Sentirse desorientado o como "ido" & .672 & \\
Dificultades para prestar atención y concentrarse & .577 & \\
Perderse en lugares conocidos & .534 & \\
Sentirse nervioso, angustiado & & .764 \\
Sentirse deprimido, triste, tenso, indefenso o desesperado & & .712 \\
Tener ideas que no puede quitarse de la cabeza & & .629 \\
Sentirse observado & & .511 \\
Llorar o reír sin motivo & & .483 \\
Salirse de las casillas en algunas situaciones & 3.84 & .446 \\
\hline Autovalor & 32 & 1.42 \\
\% explicado de la varianza & & 11.8 \\
\hline
\end{tabular}

El análisis de los estilos de vida (ver Tabla 5), arrojo un total de 3 factores que explican un $43 \%$ de la varianza. En el Factor 1 explica un 19,7\% de la varianza y en él saturaron todos los ítems relacionados a la ingesta de alcohol y el grado de actividad física. El Factor 2 se denominó Dietas prescriptas ya que en él saturan únicamente los ítems referidos a prescripciones dietéticas o nutricionales. En el Factor 3 saturaron los ítems relacionados al Hábito de fumar, este ítem explicó un 9\% de la varianza total.

En relación al Nivel funcional (ver Tabla 6), la solución factorial encontrada explica un 66,3\% de la varianza. El Factor 1 explica un $49 \%$ de la varianza total y agrupa los ítems relacionados a las Actividades instrumentales de la vida diaria. También saturó en dicho factor el ítem referido a la valoración subjetiva de la autonomía y algunos ítems relacionados a las Actividades de la vida diaria básicas como bañarse y caminar. En el Factor 2 saturaron los ítem referidos a las Actividades básicas de la vida diaria, es decir aquellas que en el curso del deterioro son las últimas en perderse pero cuya pérdida sume al sujeto en una dependencia total. El Factor 3 explicó un 6,8\% de la varianza y en el saturan los tres ítems relacionados a la Valoración subjetiva del nivel funcional y su inclusión se debió a que, su 
autovalor es mayor que 1 (Método de Kaiser), es conceptualmente distinto a los restante y tiene una entidad diferencial, ya que en el sólo saturaron los ítems vinculados a la evaluación subjetiva.

Tabla 5.

Matriz de factores rotados de los ítems de la sub-escala estilos de vida

\begin{tabular}{lccc}
\hline Ítems & Factor 1 & Factor 2 & Factor 3 \\
\hline Tomar vino o cerveza & .735 & & \\
Licores, bebida blanca & .702 & & \\
Tomar vermouth, jerez & .677 & & \\
Ocupación del tiempo libre & .670 & & \\
Sidra o champaña & .607 & & \\
Limitar grasas & & .778 & \\
Limitar sal & & .718 & \\
Limitar azúcar & & .659 & \\
Cantidad de cigarrillos diarios & & -.635 \\
Habito de fumar & & & -.523 \\
\hline Autovalor & 2.56 & 1.82 & 1.19 \\
\% explicado de la varianza & 19,7 & 14 & 9,2 \\
\hline
\end{tabular}

Tabla 6.

Matriz de factores rotados de los ítems que componen al sub-escala nivel funcional

\begin{tabular}{lccc}
\hline Ítems & Factor 1 & Factor 2 & Factor 3 \\
\hline Ir de compras & .856 & & \\
Usar el transporte & .840 & & \\
Preparar comidas & .836 & \\
Tareas domésticas & .823 & \\
Manejar dinero & .752 & & \\
Auto-validez & -.685 & & \\
Administrarse medicinas & .566 & .494 & \\
Usar el teléfono & .447 & .421 & \\
Vestirse & & .859 & \\
Cuidar aspecto físico & & .853 & \\
Ir al baño & & .845 & \\
Comer solo & & .770 & \\
Caminar (trasladarse) & .455 & .717 & \\
Bañarse & .584 & .675 & \\
N. funcional evaluación a futuro & & & .748 \\
N. funcional evaluación retrospectiva & & & -.698 \\
\hline Autovalor & 8.32 & 1.78 & 1.16 \\
\% explicado de la varianza & 49 & 10.5 & 6.8 \\
\hline
\end{tabular}


La solución factorial encontrada para la subescala Ocio y uso del tiempo (ver Tabla 7), consta de dos factores que explican el $45 \%$ de la varianza total. El primer factor se denominó Ocio Activo y Social ya que en él saturaron actividades como hacer excursiones, viajar, ir de compras, realizar trámites y gestiones, cuidar niños, visitar amigos y tareas del hogar, entre otras. El Factor 2 explica un $6.5 \%$ de la varianza total y se denominó Ocio sedentario y solitario ya que en este tipo de actividades no se requiere ningún esfuerzo especial, son actividades sedentarias y solitarias que no implican ningún cambio de actividad asociada al uso de la mayor disposición de tiempo libre.

Tabla 7.

Matriz de factores rotados de los ítems de al sub-escala ocio y uso del tiempo

\begin{tabular}{|c|c|c|}
\hline Ítems & Factor 1 & Factor 2 \\
\hline Ir de compras & .834 & \\
\hline Hacer tareas del hogar & .817 & \\
\hline Ir de compras (satisfacción) & .804 & \\
\hline Hacer gestiones y trámites & .733 & \\
\hline Satisfacción con las tareas del hogar & .731 & \\
\hline Hacer gestiones y trámites (satisfacción) & .719 & \\
\hline Nueva actividad luego de 65 años & -.718 & \\
\hline Viajar (satisfacción) & 677 & \\
\hline Ir a algún espectáculo (satisfacción) & 672 & \\
\hline Hacer excursiones (satisfacción) & .672 & \\
\hline Viajar & .615 & \\
\hline Ir la iglesia (satisfacción) & .607 & \\
\hline Hacer excursiones & .605 & \\
\hline Ir a algún espectáculo & .569 & \\
\hline Cuidar niños & .541 & \\
\hline Visitar amigos o parientes & .496 & \\
\hline Cuidar niños (satisfacción) & .470 & \\
\hline Hacer manualidades & .464 & \\
\hline Hacer manualidades (satisfacción) & .441 & \\
\hline Ver TV & & .766 \\
\hline Ver TV (satisfacción) & & .756 \\
\hline Leer diario (satisfacción) & & .590 \\
\hline Leer el diario & & .586 \\
\hline Escuchar radio (satisfacción) & & .576 \\
\hline Escuchar radio & & .487 \\
\hline Visitar amigos (satisfacción) & .470 & .476 \\
\hline Jugar a las cartas & & .462 \\
\hline Caminar (satisfacción) & .424 & .440 \\
\hline Jugar a las cartas & & .429 \\
\hline Autovalor & 7.45 & 1.34 \\
\hline$\%$ explicado de la varianza & 39 & 6,5 \\
\hline
\end{tabular}


El análisis del Apoyo social (ver Tabla 8) arrojó una solución que explica el $51 \%$ de la varianza. El Factor 1, Satisfacción familiar, explicó un 15\% de la varianza total y en el saturan los indicadores del grado de satisfacción con las relaciones mantenidas con hijos y nietos. El FACTOR 2 explica un 11\% de la varianza y en él saturan los ítems relacionados a la Frecuencia de contactos, que representa la frecuencia de visitas de hijos, nietos y otros familiares. En este factor también saturó el número de miembros que viven en el hogar y el mismo. Este último ítem, si bien no representa visitas, implica una red social más amplia que intensifica las interacciones con diferentes personas. El Factor 3 se denominó Relaciones íntimas ya que en este factor saturan ítems relacionados con esto. Este factor explica un 9,4\% de la varianza total. En el Factor 4, Relaciones amistosas, saturaron todos los ítems referidos al grado de satisfacción con amigos, familiares cercanos y vecinos. Este factor explicó casi un $8 \%$ de la varianza. En el factor 5 saturaron solo los ítems referidos a la Frecuencia y satisfacción sexual. Dicho factor explica un $7 \%$ de la varianza total.

Tabla 8.

Matriz de factores rotados de los ítems que componen la sub-escala integración social

\begin{tabular}{|c|c|c|c|c|c|}
\hline Ítems & Factor 1 & Factor 2 & Factor 3 & Factor 4 & Factor 5 \\
\hline Satisfacción con hijos & .817 & & & & \\
\hline Satisfacción con los nietos & .793 & & & & \\
\hline Visita a amigos & & .669 & & & \\
\hline ¿Con quién vive $\mathrm{Ud}$ ? & & -.669 & & & \\
\hline Visita a nietos & .565 & .620 & & & \\
\hline Visita a hijos & .490 & .579 & & & \\
\hline Presencia de relaciones sexuales & & & -.775 & & \\
\hline Modificación de apetencia sexual & & & .739 & & \\
\hline Edad del cónyuge & & & -.442 & & \\
\hline Satisfacción con el cónyuge & & & .427 & & \\
\hline Satisfacción con otros familiares & & & & .627 & \\
\hline Satisfacción con vecinos & & & .420 & .565 & \\
\hline Satisfacción con amigos & & & & .524 & \\
\hline $\begin{array}{l}\text { Grado de satisfacción de relaciones } \\
\text { sexuales }\end{array}$ & & & & & .731 \\
\hline Frecuencia de relaciones sexuales & & & & & .725 \\
\hline Autovalor & 2.56 & 1.86 & 1.59 & 1.33 & 1.23 \\
\hline$\%$ explicado de la varianza & 15.1 & 11 & 9.4 & 7.8 & 7.3 \\
\hline
\end{tabular}

El análisis de la dimensión Satisfacción vital (ver Tabla 9) arrojó una solución factorial de dos factores que explican un $42,6 \%$ de la varianza total. El Factor 1 fue denominado Posición depresiva ya que los elementos que saturan sobre él reflejan una 
posición pesimista y negativa frente al paso de los años. Este factor por separado explica el $32 \%$ de la varianza. El Factor 2 refleja una disposición de Irritabilidad, ya que refleja la postura de los ancianos que se alteran fácilmente y explica el 10,8\% de la varianza.

\section{Tabla 9.}

Matriz de factores rotados de los ítems de la sub-escala satisfacción vital

\begin{tabular}{lcc}
\hline Ítems & Factor 1 & Factor 2 \\
\hline Menos útil al ser mayor & .642 & \\
A veces siento que la vida no merece la pena ser vivida. & .636 & \\
Ahora soy tan feliz como cuando era joven. & -.635 & \\
Según me voy haciendo mayor, me siento más solo. & .618 & \\
Siento que según me voy haciendo mayor soy menos útil. & .598 & \\
A medida que me voy haciendo mayor, se ponen las cosas peor & .548 & .372 \\
para mí. & .476 & .392 \\
Tengo muchas razones por las que estar triste. & .469 & .315 \\
A veces estoy tan preocupado que no puedo dormir. & & .732 \\
Me siento más irritable que antes. & & .725 \\
Me altero o disgusto fácilmente. & & .656 \\
Ahora me molestan más las cosas pequeñas que antes. & .654 \\
Me tomo las cosas a pecho. & & .412 \\
La mayor parte del tiempo la vida es dura y difícil. & 4.12 & 1.40 \\
\hline Autovalor & 31,8 & 10,8 \\
\hline \% explicado de la varianza & & \\
\hline
\end{tabular}

Mediante el análisis factorial del CBCV, incluyendo todas sus sub-escalas, se extrajeron 3 factores con el método de extracción de factores principales con rotación Varimax, los cuales explicaron un $44,3 \%$ de la varianza total.

En el Factor 1, que se denominó Actividad / Autonomía saturaron todas las subescalas referidas al uso del tiempo. También saturó sobre este factor el grado de dificultad experimentado por los ancianos en la realización de diferentes actividades de la vida diaria (básicas e instrumentales) que se encuentran comprometidas en el mantenimiento de su autonomía e independencia. Finalmente saturó sobre este mismo factor sólo la frecuencia de visitas realizadas a amigos y parientes. Todas estas actividades se encuentran determinadas por el grado de independencia y movilidad que tenga el anciano. El Factor 2 (Salud Y Bienestar), incluyó las escalas referidas a la salud, tanto física como psíquica. En el Factor 3, denominado Calidad Socio-Ambiental, saturaron todos los ítems referidos a las comodidades y satisfacción con la vivienda particular, así como el nivel de ingresos de los ancianos. 
Tabla 10

Matriz de factores rotados de las diferentes sub-escalas del CBCV

\begin{tabular}{lccc}
\hline Ítems & Factor 1 & Factor 2 & Factor 3 \\
\hline Satisfacción con las actividades del tiempo libre & .917 & & \\
Frecuencia de las actividades del tiempo libre & .907 & & \\
Dificultades AVD básicas e instrumentales & -.824 & & .414 \\
Evaluación subjetiva del nivel funcional. & .489 & & \\
Frecuencia de contactos sociales & .475 & & \\
Trastornos crónicos de salud & & .825 & \\
Dolores recientes & & .823 & \\
Salud psíquica & & .700 & \\
Ingesta de medicamento & -.656 & \\
Satisfacción vital & & .618 & \\
Salud subjetiva & & .509 &. .662 \\
Comodidades en la vivienda. & & & .567 \\
Satisfacción con la vivienda & & & .498 \\
Satisfacción con el barrio & & & .464 \\
Satisfacción con las relaciones sociales & & & \\
Nivel de ingresos & 4.48 & 3.03 & 7.4 \\
\hline Autovalor & & 14.5 & \\
\% explicado de la varianza & & & \\
\hline
\end{tabular}

\subsection{Análisis de confiabilidad.}

El coeficiente Alpha de las dimensiones estado mental, salud objetiva, psíquica, habilidades funcionales, uso del tiempo y satisfacción vital supera el $0.75 \mathrm{y}$ el de Ambiente obtuvo un $\alpha=.68$. Esto demuestra una buena consistencia interna del CBCV.

Tabla 11.

Consistencia interna de las sub-escalas del Cuestionario Breve de Calidad de Vida

\begin{tabular}{lccc}
\hline Dimensiones & $\begin{array}{c}\mathbf{N}^{\mathbf{0}} \mathbf{d e} \\
\text { ítems }\end{array}$ & $\mathbf{N}$ & Alpha \\
\hline Salud Sujetiva & 2 & 471 & .82 \\
Salud Objetiva (dolores recientes, visitas al médicos y días de & 25 & 433 & .94 \\
cama) & 8 & 502 & .88 \\
Salud Psíquica & 6 & 97 & .76 \\
Apoyo social (frecuencia de visitas de hijos, nietos, familiares y & 6 & & .93 \\
amigos) & 11 & 476 & .93 \\
Habilidades Funcionales: dificultad & 13 & 453 & .89 \\
Actividad: frecuencia & 18 & 457 & .68 \\
Ambiente & 5 & 443 & .90 \\
Satisfacción vital & 88 & & \\
\hline Número total de ítem & & & \\
\hline
\end{tabular}




\section{Discusión.}

El objetivo de este estudio fue elaborar y analizar las propiedades psicométricas de un instrumento que permitiera la evaluación de las diversas dimensiones de la calidad de vida. Disponer de un instrumento de estas características es de suma importancia para poder evaluar la calidad de vida de un sujeto o grupo de personas en vistas a diseñar o evaluar las intervenciones dirigidas a los adultos mayores.

Los resultados mostraron al CBCV como un instrumento válido y fiable para medir tales factores. Sin embargo, en función de la importancia del concepto de Calidad de Vida en adultos mayores, la valdación de esta escala implicaría, en etapas posteriores, estudios que demuestren la estabilidad de las puntuaciones.

En los análisis factoriales realizados a cada dimensión, los ítems correspondientes al Estado mental se agruparon en dos factores que corresponden a la información actual relevante que pueden los recordar sujetos y a la información registrada de manera temprana, las cuales presentan mecanismos diferentes de almacenamiento y recuperación.

En la dimensión Salud subjetiva pudieron identificarse diferencias entre la valoración actual del estado de salud de los ancianos y la evolución temporal de la misma que considera la expectativa de funcionamiento futuro y parámetros comparativos con otros individuos de su misma edad.

El análisis factorial de sub-escala correspondiente al Padecimiento de enfermedades e ingesta de medicamentos mostró tres factores diferentes referidos a problemas osteoarticulares, trastornos del sueño y problemas bronquio-respiratorios.

En relación a la dimensión Salud psíquica pueden observarse dos factores claramente diferenciados, uno que representa problemas mnésicos (memoria y atención) y otro vinculado a malestares de tipo emocionales y subjetivos específicos (depresión, angustia, nerviosismo, etc.).

La dimensión Estilos de vida presentó diferencias entre la ingesta de alcohol, dietas prescriptas y el hábito de fumar, todos ellas constituyen factores de riesgo en relación a la calidad de vida de los mayores.

En la dimensión Nivel funcional aparecieron dos grupos de ítems que reflejan conceptos generales básicos en la evaluación geronto-geriátrica: la autonomía, distinguiéndose las actividades básicas de la vida diaria de las instrumentales, y la valoración subjetiva del nivel funcional propio. 
En el análisis factorial de la dimensión Ocio y el uso del tiempo libre mostró una clara diferencia entre las actividades que implican salir de la casa y en algunas de ellas involucrarse con otras personas, en contraposición a actividades de tipo sedentarias y solitarias. También presentaron diferencias en cuanto al nivel de compromiso físico involucrado en tales actividades.

Así también, en la dimensión Apoyo social aparecieron factores que distinguen la frecuencia de las relaciones y su distinción según el grado de intimidad de las mismas.

La dimensión Calidad ambiental mostró diferencias referidas a la calidad de la propia vivienda y la conformidad con el propio barrio.

Finalmente en relación a la Satisfacción vital, aparecieron dos factores que refieren a variables clásicas de disposición hacia el propio envejecimiento y muestran diferencias centrales en el afrontamiento de la vida; una posición depresiva, pesimista y negativa frente al paso de los años y una disposición de irritabilidad.

El análisis factorial del cuestionario completo muestra claramente la multidimensionalidad del constructo tal como lo plantea Lawton (1991) en su definición de la misma. La Calidad de Vida ha probado ser, por tanto, una evaluación multidimensional, subjetiva y objetiva, del sistema persona-ambiente; sistema que supone la competencia conductual, entendiendo a ésta como un continuo de manifestaciones conductuales de diferentes niveles: biológico, funcional, cognitivo, personal, social y recreativo.

Por lo tanto, puede decirse que no existe un sólo constructo asociado a la calidad de vida, sino que su definición implica ciertas propiedades inherentes a distintos ámbitos importantes en la vida de las personas -su salud, sus relaciones sociales, su estatus socio económico, el uso de su tiempo, etcétera- que nos permitan apreciar diferencias y establecer comparaciones, tanto entre los individuos, cuanto entre diferentes momentos y condiciones de la vida de una misma persona.

El análisis factorial realizado a los fines de acercar evidencias sobre la validez de constructo del cuestionario evidenció cuatro conceptos básicos vinculados a la calidad de vida que subyacen a todo el instrumento: la autonomía y nivel de actividad, la salud y el bienestar psicológicos y los componentes socio ambientales vinculados a la satisfacción con la vivienda o residencia. Estos tres factores encontrados y los ítems que saturaron en ellos permiten redefinir el concepto de calidad no sólo como multidimensional sino también como concepto cuyas dimensiones se encuentra interrelacionadas. 
En resumen, los factores encontrados en este último análisis, vinculan la estructura factorial, a dimensiones conceptuales encontradas en la mayoría de los estudios, tales como la salud, la satisfacción vital, el uso del tiempo y las relaciones sociales.

La solución factorial encontrada es comparables con la obtenida por Fernández Ballesteros y sus colaboradores (1996) ya que ambas evidencian que en la evaluación de la calidad de vida en mayores, si bien el concepto es multidimensional, las esferas principales de evaluación son: la salud, el apoyo social y la autonomía y nivel de actividad de los ancianos. Estas dimensiones no pueden dejar de evaluarse a la hora de tener una valoración global de la vida de los mayores y que aspire a la exhaustividad.

\section{Referencias}

Birren J. E. y Diekman L. (1991). Concepts and content of quality of life in the later years: an overview en The Concept and Measurement of Quality of Life in the Frail Elderly. Birren, J.E.; Lubben, J.E.; Chichowlas Rowe, J.y Deutchman, D.E. Londres: Academic Press.

Comisión Económica para América Latina y el Caribe. Centro Latinoamericano y Caribeño de Demografía [CEPAL-CELADE]. (2006). Manual sobre indicadores de calidad de vida en la vejez. Santiago de Chile. Extraído el 2 de febrero de http://www.eclac.cl/publicaciones/xml/0/28240/W113_1.pdf

Fernández Ballesteros, R., Zamarrón, M. y Maciá, A. (1996). Calidad de vida en los distintos contextos. Universidad Autónoma de Madrid, Ministerio de Trabajo y Asuntos Sociales, Instituto nacional de Servicios Sociales (INSERSO).

Kaplan, R. M. y Bush, J. W. (1982). The quality of well-being (QWB) scale. Health and Psychology, 61-80.

Lawton M. P. (1991). A Multidimensional View of Quality of Life in Frail Elders en The Concept and Measurement of Quality of Life in the Frail Elderly de Birren, J.E.; Lubben, J.E.; Chichowlas Rowe, J.; Deutchman, D.E. Academic Press.

Organización Mundial de la Salud [OMS]. (1994). Calidad de vida. Extraído el 24 de octubre de 2005 de http://encyclopedie-es.snyke.com/articles/calidad_de_vida.html.

Urrutia, A. (2006). Dimensiones del concepto de Calidad de Vida en ancianos. Elaboración de un instrumento multivariable para su evaluación. Tesis de Doctorado. Facultad de Psicología, Universidad Nacional de Córdoba.

Yanguas Lezaun, J. J. (2006). Análisis de la calidad de vida relacionada con la salud en la vejez desde una perspectiva multidimensional. Instituto de Mayores y Servicios Sociales (IMSERSO). Artegraf SA, Madrid. 